Stomatologie 2015 $\cdot 112: 57-58$ DOI 10.1007/s00715-015-0033-x Online publiziert: 17. April 2015 ○) Springer-Verlag Wien 2015

\section{Irmgard Simma}

Praxis für ganzheitliche Zahnheilkunde und Kieferorthopädie, Zahn-, Mund- und Kieferheilkunde, Bregenz, Österreich

\title{
Ganzheitliche Zahnmedizin
}

Liebe Kolleginnen und Kollegen,

ich freue mich im Namen der Gesellschaft für ganzheitliche Zahnheilkunde das Themenheft „Ganzheitliche Zahnmedizin“ der Zeitschrift stomatologie präsentieren zu dürfen.

Die Gesellschaft für ganzheitliche Zahnheilkunde ist eine Sektion der Österreichischen Gesellschaft für Zahn-, Mund- und Kieferheilkunde (ÖGZMK). Sie besteht seit 1997 und wurde anlässlich des Zahnärztekongresses an der Universität Wien von Univ.-Prof. Slavicek, dem damaligen Präsidenten der ÖGZMK, und Dr. Simma als Arbeitsgemeinschaft gegründet.

Die Entwicklung der ganzheitlichen Zahnheilkunde ist das Ergebnis des zunehmenden Bestrebens, über die offizielle Schulmedizin hinaus auch komplementäre und naturheilkundliche Ideen und Methoden in die Diagnose und Therapie zu integrieren. Mit dem anspruchsvollen Titel "ganzheitlich " ist immer auch die Verpflichtung verbunden, solche Methoden im Sinne einer evidenzbasierten Medizin kritisch zu prüfen. Als Evidenz ist aber auch das positive Feedback von Hunderten Fachkollegen und Patienten zu werten, das in seiner Bedeutung den Ergebnissen von Labor- und Tierversuchen gleichzusetzen ist.

Die Tätigkeit des Zahnarztes verlangt neben dem hohen Pflichtbewusstsein die Fähigkeit, Kompromisse einzugehen, aber auch Intuition und Kreativität in der Erstellung von Diagnosen und Therapien. Auf diese Weise wird nicht nur eine nachhaltige Wirksamkeit erzielt, sondern auch die Möglichkeit für die Gewinnung neuer Erkenntnisse geschaffen.

Viele der ganzheitlichen bzw. komplementären Methoden wurden von der Schulmedizin über Jahrzehnte nicht ernst genommen. Inzwischen hat sich die Wir- kungsweise mancher Methoden wissenschaftlich verifizieren lassen, so z. B. die Wirkung der „Heilinjektion“, die wesentlich auf dem antiinflammatorischen Effekt von Anästhetika (Procain) beruht. Ebenso konnte die analgetische Wirkung der Akupunktur wissenschaftlich nachgewiesen werden (Freisetzung von Endorphinen).

Die erhebliche Zunahme funktioneller Beschwerdebilder - oftmals mit psychischem Hintergrund - hat zu einem gesteigerten Einsatz komplementärer Methoden geführt. Die funktionellen Vernetzungen und Wechselwirkungen überschreiten die Grenzen von Fachdisziplinen, so auch die der Zahn-Kiefer-Heilkunde. Beispielsweise ist die Beteiligung der Halswirbelsäule bei chronischen Belastungen im Zahn-Kiefer-Bereich bekannt, die erfahrungsgemäß optimal und nachhaltig mittels Osteopathie, Neuraltherapie oder Akupunktur therapiert werden kann.

Ferner hat die Formulierung der Grundregulation nach Pischinger zur Etablierung verschiedener Modelle der Regulation bzw. der Regulationspathologie geführt.

$\nabla$

Die Matrix dient als schul- und komplementärmedizinisches Erklärungsmodell für Diagnose und Therapie.

Es kann postuliert werden, dass aus rein zahnmedizinischer Sicht die Positionierung des Unterkiefers und damit des Kiefergelenks in der Okklusion eine zentrale Rolle im Gesamtsystem spielt. Sie beeinflusst die Haltung und Körperstatik und konstituiert mit der Psychosomatik (5-Elemente-Lehre) ein kompatibles Erklärungsmodell, das als „Verlaufskontrolle“ ein zusätzlicher diagnostischer und therapeutischer Wegweiser sein kann. In diesem Sinne wurde bei vielen ener- getischen Testmethoden die Wichtigkeit und die zentrale Rolle der Zahnmedizin (Störfeld Kiefergelenk) evaluiert.

Das Konzept der Gesellschaft für ganzheitliche Zahnheilkunde (s. - Infobox) umfasst daher nicht nur die schulmedizinische Diagnostik und Therapie, (wie die instrumentelle und manuelle Funktionsdiagnostik), sondern auch diverse andere Methoden, um Struktur und Funktion zu erfassen. Hierbei ist die Osteopathie als strukturell-funktionales Modell sehr hilfreich. Auf der chemischen, psychischen und bioinformativen Ebene gehören Lymphtherapien, Ernährung, Homöopathie, psychisch harmonisierende Therapien und die Mundakupunktur zur Domäne des Zahnarztes.

Gerade über die Mundakupunktur erfolgt eine gesunde Entspannung der Kaumuskulatur, sodass das Kausystem als Stressbearbeitungsorgan entlastet wird und diverse CMD-Symptomatiken harmonisiert werden.

Eine interessante Lektüre wünscht Ihnen

DDr. Irmgard Simma

Präsidentin für ganzheitliche ZHK

\section{Korrespondenzadresse}

Univ.-Lektorin. DDr. I. Simma

Praxis für ganzheitliche Zahnheilkunde und Kieferorthopädie, Zahn-, Mundund Kieferheilkunde Arlbergstr. 139, 6900 Bregenz dri.simma@aon.at 


\section{Infobox Konzept der Gesellschaft für ganzheitliche Zahnheilkunde}

Ganzheitliche Zahnheilkunde beschäftigt sich mit den multifunktionellen Bezügen des stomatognathen Systems zum Gesamtorganismus und zur Umwelt. Symptome des Kausystems werden nicht isoliert betrachtet, sondern mit dem allgemeinen Erscheinungsbild des Menschen und seiner Befindlichkeit in Beziehung gebracht. Aus Konzepten, denen somatische, psychische, psychosoziale, biokybernetische und informative Vernetzungen zugrunde liegen, wurde das Konzept der Gesellschaft für ganzheitliche Zahnheilkunde praxisbezogen erarbeitet. Es kann über aktuelle Belastungen, Herde und Störfelder, aber v. a. über die Regulationsfähigkeit des Patienten informieren und verfügt über Methoden, die das Kausystem als Stressbeantwortungsorgan entlasten. Des Weiteren kann es prophylaktisch und begleitend zahnärztliche Behandlungen erleichtern und unterstützen. Inspektion, Palpation und diverse Testmethoden dienen dazu, über Priorität und individuelle Auswahl der Behandlungsmethoden zu entscheiden. Das Konzept beruht auf 8 Punkten:

1. Ganzheitliche Anamnese

2. Panoramaröntgen

3. Äußere Inspektion

4. Innere Inspektion

5. Palpation

6. Testmethoden

7. Therapien

8. Ernährung

Zahnärztliche additive Funktionsuntersuchungen für Kinder und Erwachsene (in Kürze)

- Untersuchung der Kopf-und Gesichtssymmetrie

- Äußere Inspektion in Hinblick auf orofaziale Dyskinesien, gestörte Myofunktion, Lymphstau und Mundatmung

- Innere Inspektion: Erfassung des Status der Zähne, der Schleimhäute, der Zunge, des Lymphsystems etc.

- Extra- und intraorale Palpation - manuelle Funktionsdiagnostik: Die Kaumuskulatur extraoral und intraoral zeigt bei symmetrischer Palpation unterschiedliche Druckempfindlichkeiten (0-3). Die intraorale Palpation umfasst Muskulatur, Schleimhaut sowie Retromolaren- und Vestibulumpunkte des Mundakupunktursystems.

- Testung der intra- und extraoralen Reflexpunkte (Punkte nach Adler-Langer, Lymphbelt etc.)

\section{Fortbildungsaktivitäten/Veranstaltungen}

- Zu den genannten Themen werden sowohl Patienten, Studenten als auch Zahnärzte und Ärzte seit Gründung der Gesellschaft informiert. In diesem Zusammenhang sind insbesondere folgende Aktivitäten und Erfolge erwähnenswert: Festspielgespräche in Bregenz seit 1998, interdisziplinärer Erfahrungsaustausch zur Gesamtschau des Menschen

- Organisation der Funktionstherapien in der zahnärztlichen Praxis in Oberlech seit 1999

- Mundakupunkturkurse an der Universitätszahnklinik Wien und an den Kliniken Innsbruck, Salzburg, Bregenz, Oberlech seit 1998

- Lehrauftrag an den österreichischen Universitätszahnkliniken seit 1998 in Wien, seit 2008 in Graz

- Lehrauftrag an der Akademie für Ganzheitsmedizin GAMED, Wien, seit 2003

- Lehrauftrag Ringvorlesung für Komplementärmedizin an der Medizinischen Universität Wien:„Ganzheitliche Funktionstherapien in der Zahnheilkunde ${ }^{\prime \prime}$, Mundakupunktur und Mikrosysteme" seit 2005

- Organisation des komplementären Teils der österreichischen Zahnärztekongresse seit 1996

- Vortragstätigkeit im In- und Ausland auf internationalen Kongressen

\section{Studien und Publikationen}

- 1994-2004: Durchführung einer Mundakupunkturstudie an der Universitätszahnklinik in Wien in Zusammenarbeit mit E. Piehslinger, J. Gleditsch und I. Simma-Kletschka

- Zahlreiche Publikationen und Kongressbände zur ganzheitlichen Zahnheilkunde seit 1996

- Betreuung von Diplomarbeiten

- Diverse Medienberichte in ORF, Radio Vorarlberg, Ö1 und den Vorarlberger Nachrichten

\section{Awards}

- 2008: Slavicek-Preis gemeinsam mit M. Schmid-Schwap, E. Piehslinger, J. Gleditsch

- 2008: ICMART Budapest Scientific Acupuncture Award (1. Platz)

- 2009: Bischko-Preis, 3. Johannes-Bischko-Symposium Wien (1. Platz)

\section{Kooperationen}

- Gesellschaft für ganzheitliche Zahnmedizin in Deutschland und Schweiz (GZM, SGZM)

- Mitglied des Dachverbandes österreichischer Ärztinnen und Ärzte für Ganzheitsmedizin: Ziel dieser Mitgliedschaft ist es, sowohl Ganzheitsmediziner anderer Fachgebiete als auch Patienten zu informieren.

- GAMED

- Akupunkturgesellschaften ÖGA, DGfAN, DÄGFA

- Ganzheitliche Zahnheilkunde (ARGE des ZIV)

Studenten, Zahnärzte und Ärzte sind herzlich eingeladen, sich bei unseren Veranstaltungen zu informieren, Ideen und Fragen einzubringen und v. a., sie auch mitzugestalten. 